\section{Cahiers de Narratologie}

Analyse et théorie narratives

$24 \mid 2013$

Avant-gardes et littérature narrative

\title{
Bibliographie : Les avant-gardes historiques et les écritures narratives
}

\section{(2) OpenEdition}

Journals

Édition électronique

URL : http://journals.openedition.org/narratologie/6680

DOI : 10.4000/narratologie.6680

ISSN : 1765-307X

Éditeur

LIRCES

Référence électronique

"Bibliographie: Les avant-gardes historiques et les écritures narratives », Cahiers de Narratologie [En

ligne], 24 | 2013, mis en ligne le 17 septembre 2013, consulté le 19 avril 2019. URL : http://

journals.openedition.org/narratologie/6680; DOI : 10.4000/narratologie.6680

Ce document a été généré automatiquement le 19 avril 2019

Article L.111-1 du Code de la propriété intellectuelle. 


\section{Bibliographie: Les avant-gardes historiques et les écritures narratives}

1 - Futurismo e letteratura, L'Illuminista. Rivista di cultura contemporanea (Roma), n²7, settembre/dicembre 2009

2 - Albert, Mechthild, « Ramón Gómez de la Serna. El novelista (1925) », in Spanische Romane des 20. Jahrhunderts in Einzeldarstellungen, Hg. Ralf Junkerjürgen, Berlin, Erich Schmidt, 2010, p. 65-79

3 - Arnold, Armin, Prosa des Expressionismus. Herkunft, Analyse, Inventar, Stuttgart: W. Kohlhammer, 1972

4 - Asholt Wolfgang et Fähnders, Walter (Hg.), Der Blick vom Wolkenkratzer : Avantgarde, Avantgarde-Kritik, Avantgarde- Forschung, Amsterdam, Rodopi, 2000

5 - Bergeron, Patrick, « Céleste Ugolin (1926) de Georges Ribemont-Dessaignes et l'influence d'Alfred Jarry dans le roman ", Études littéraires, vol. 36, n 3, 2005, p. 15-41

6 - Boucharenc, Myriam et Rubio, Emmanuel (éd.), Réinventer le roman dans les années vingt, Villeneuve d'Ascq, Presses de l'Université Charles-de-Gaulle - Lille 3, 2010

7 - Bradbury, Malcolm et McFarlane, James, Modernism. 1890-1930, New York, Penguin, 1976

8 - Bruner, Jeffrey, «La importancia de Ramón Gómez de la Serna en la novela española moderna: El caso de El novelista: 1988-1989 ", in Selected Proceedings of the Pennsylvania Foreign Language Conference. Martín, Gregorio C. (Hg.), Pittsburgh, Dept. of Mod. Langs., Duquesne Univ., p. 60-67.

9 - Cannistraro, Philip V., La fabbrica del consenso. Fascismo e mass media, Bari, Laterza, 1975

10 - Carlat, Dominique, «La Voix du romanesque poétique: "la force soudaine d'une reconstitution" ", in Patiences et silences de Philippe Soupault, Jacqueline Chénieux-Gendron dir., Paris, L'Harmattan, 2000, p. 145-157

11 - Cescutti, Tatiana, Les Origines mythiques du futurisme. Marinetti poète symboliste, Paris, PUPS, 2008

12 - Chartier, Jean, «Philippe Soupault. Le choix de la prose dans la décennie 1917-1927 », Mélusine, n 15, 1995, p. 293-296 
13 - Chénieux-Gendron, Jacqueline, Le Surréalisme et le roman, Lausanne, L’Âge d'Homme, 1983

14 - Cigliana, Simona, Futurismo esoterico, Napoli, Liguori, 2002

15 - Collani, Tania, Le Merveilleux dans la prose surréaliste européenne, Paris, Hermann, 2010

16 - Contarini, Silvia, « Il Futurismo e il romanzo », Narrativa, n. 9, février 1996

17 - Contarini, S. et Cardini, K. (éd.), Le Futurisme et les Avant-Gardes littéraires et artistiques au début du XXe siècle, Nantes, CRINI, 2002.

18 - Coste, Grégory, «Le récit d'avant-garde espagnol: les avatars d'une tentative de renouvellement sur les marges du roman ", Pandora, $n^{\circ} 9,2009$, p. 235-252

19 - Id., «Subversión del discurso mitológico y renovación del género novelístico en la narrativa de vanguardia (1926-1934) ", in Myth and Subversion in the Contemporary Novel, José Manuel Losada and Marta Guirao (éd.), Cambridge Scholars Publishing, 2012, p. 41-52

20 - D'Ascia, Luca, « Il romanzo futurista : estetica dell'immagine e civiltà di massa », in Arte d'avanguardia e società. L'esperienza futurista nel pensiero sociale e culturale contemporaneo, Ilaria Ricconi (éd.), Rome, L’Albatros, 2006

21 - De Donato, G. e Gazzola Stacchini, V. (a cura di), I best seller del ventennio. Il regime e il libro di massa, Roma, Editori Riuniti, 1991

22 - Del Pino, Juan Manuel, Montajes y fragmentos: una aproximación a la narrativa española de vanguardia, Amsterdam, Rodopi, 1995

23 - De Maria, Luciano, " Postfazione », in F.T. Marinetti, Teoria e invenzione futurista (1968), Milano, Mondadori - I Meridiani, 1983

24 - Devésa, Jean-Michel, René Crevel et le roman, Editions Rodopi, 2004

25 - Doane, Janice L., Silence and Narrative: The Early Novels of Gertrude Stein, Westport, CT, Greenwood Press, 1986

26 - Dufour, Catherine, " Mais où est passé le roman dada? ", in Réinventer le roman dans les années vingt, Myriam Boucharenc et Emmanuel Rubio éd., Villeneuve d'Ascq, Presses de l'Université Charles-de-Gaulle - Lille 3, 2010, p. 75-92

27 - Dupuis, Michel, «Le roman », in Les Avant-gardes littéraires du XXe siècle, Jean Weisgerber (éd.), publication du Centre d'étude des avant-gardes littéraires de l'université de Bruxelles / coll. « Comparative History of Literatures in European Languages », Budapest, Akadémiai Kiadó, 1984-1986, vol. 1, p. 850-877

28 - Edwards, Paul, Soleil noir, photographie et littérature des origines au surréalisme, Rennes, Presses universitaires de Rennes, 2008

29 - Fernández Cifuentes, Luis, Teoría y mercado de la novela en España: del 98 a la República, Madrid, Gredos, 1982

30 - Fernández Utrera, María Soledad, Visiones de estereoscopio. Paradigma de hibridación en el arte y la narrativa de la vanguardia española, Chapel Hill, The University of North Carolina Press, Studies in the Romances Languages and Literatures, 2001

31 - Gahl, Peter, « Das intensive Leben der Postavantgarde. Metanarrative Komik in Massimo Bontempellis La vita intensa ", in Avantgarde und Komik,. hrg. v. L. Scherer, R. Lohse. Amsterdam, 2004, p. 143-157

32 - Garnier, Pierre et Ilse, «La prose expressionniste », in L’Expressionnisme allemand, Paris, A. Silvaire, 1962, rééd. 1979 
33 - Giocondi, Michele, Lettori in camicia nera. Narrativa di successo nell'Italia fascista, Messina-Firenze, D'Anna, 1978

34 - Glicksohn, Jean-Michel, «La prose expressionniste », in L'Expressionnisme littéraire, Paris, PUF, 1990, ch. 4, p. 95-116

35 - González-Gerth, Miguel, A labyrinth of imagery: Ramón Gómez de la Serna's "novelas de la nebulosa », London, Tamesis Books Ltd., Colección Támesis, Serie A, Monografias, 120, 1986

36 - Helmich, Werner, «Le lieu historique du novelista ramonien dans la perspective du roman autoréférentiel contemporain ", in Ramón Gómez de la Serna, Evelyne MartinHernández (éd.), Clermont-Ferrand, Univ. Blaise-Pascal, 1999, p. 157-189

37 - Hernando, Miguel Ángel, Prosa vanguardista en la generación del 27, Madrid, Prensa Española, 1975

38 - Hubner-Bayle, Corinne, Romans et contes de Pierre Reverdy: une poétique de la marge : étude sur « Le voleur de Talan » (1917), «La peau de l'homme » (1926), « Risques et périls (1930), Paris, H. Champion, 1993

39 - Jaccard, Jean-Philippe, Daniil Harms et la fin de l'avant-garde russe, Bern / Frankfurt a.Main / New York / Paris, Wien, Peter Lang, 1991

40 - Jens, Inge, Die expressionistische Novelle: Studien zu ihrer Entwicklung, Tübingen, Attempto, 1997

41 - Kahler, Erich von, « Die Prosa des Expressionismus », Der deutsche Expressionismus. Formen und Gestalten, hrg. v. Hans Steffen, Göttingen, Vandenhœck \& Ruprecht, 1965, p. 157-178

42 - Krull, Wilhelm, Prosa des Expressionismus, Stuttgart, J. B. Metzlersche Verlagsbuchhandlung, 1984

43 - Krzywkowski, Isabelle, «Le Temps et l'Espace sont morts hier ». Les Années 1910-1920. Poésie et poétique de la première avant-garde, Paris, Éditions L'Improviste, 2006

44 - Id., «Épopée et avant-garde : l'exemple de L'Orphéide ou L'Universel Poème d'HenriMartin Barzun ", in Désirs et débris d'épopée au XXe siècle, Saulo Neiva éd., Bern, ..., Peter Lang, 2009, p. 57-74

45 - Krzywkowski, Isabelle et Millot, Cécile (dir.), Expressionnisme(s) et avant-gardes, Paris, Editions L'improviste, Collection « Les aéronautes de l'esprit », 2007

46 - Laget, Laurie-Anne, La Fabrique de l'écrivain. Les premières greguerias de Ramon Gomez de la Serna (1910-1923), Casa de Velazquez, coll. «Bibliothèque Casa de Velazque », 2012

47 - Lanne, Jean-Claude, «Les Particularités de la prose poétique chez les futuristes russes », Revue des études slaves, 1995, vol. 67, n 4, p. 605-626

48 - Le Vagueresse, Emmanuel, «Ramón ou la subversion du roman? ", in Ramón Gómez de la Serna, Evelyne Martin-Hernández dir., Cahiers de Recherches du CRLMC, ClermontFerrand, Université Blaise-Pascal, p. 179-189

49 - Losseroy, Gilles, Georges Ribemont-Dessaignes romancier, thèse de doctorat, Université de Nancy II, 1995

50 - Lough, Frank (ed.), Hacia la novela nueva. Essays on the Spanish Avant-Garde Novel, Bern, Peter Lang, 2000

51 - Magnien, Brigitte, «Crise du roman », in Temps de crise et " années folles ». Les années 20 en Espagne, Serrano, Carlos et Salaün, Serge (éd.), Paris, Presses de l'Université de Paris Sorbonne, 2002 
52 - Martini Fritz, Prosa des Expressionismus, Stuttgart, Reclam Philipp Jun., 1970

53 - Masi, A. (a cura di), Zig zag. Il romanzo futurista, Milano, Il Saggiatore, 1995, rééd. 2009

54 - McCulloch, John A., The Dilemma of Modernity. Ramón Gómez de la Serna and the Spanish Modernist Novel, New York/Bern etc., Peter Lang, 2007

55 - Meazzi, Barbara, "Un romanzo che non è un romanzo ». Le futurisme italien et la littérature narrative (1909-1929), à paraître

56 - Id., "Mario Carli et l'écriture romanesque futuriste", in Avant-gardes: frontières, mouvements, vol. II : Marges, revitalisations, Jean-Paul Aubert, Serge Milan et Jean-François Trubert (éd.), Sampzon, Delatour-France, 2012, p. 9-21

57 - Id., « Bruno Corra e il romanzo futurista », Avanguardia, XV, n. 48, 2011, p. 5-22 ;

58 - Id., « Enif Robert \& Filippo Tommaso Marinetti : Un ventre di donna e l'autobiografia futurista ", in Tempo e memoria nella lingua e nella letteratura italiana, Bart van den Bossche et al. éd. Bruxelles, AIPI, 2009, p. 19-41, http://www.infoaipi.org/attion/ascoli_vol_3.pdf

59 - Id., «Le Futurisme et le paradoxe réaliste : locomotives et chaussettes, ou du réalisme futuriste ", in Les Frontières du réalisme dans la littérature narrative du XXe siècle / The Borders of Realism in 20th Century Narrative Literature, éd. Fabry, Geneviève, Roland, Hubert, Bragard, Véronique, Jacques, Georges, Lazzarini-Dossin, Muriel ; Vanderlinden, Sonja, 2006, p. 47-58 http://hdl.handle.net/2078.1/81491

60 - Meazzi, Barbara (a cura di), L'arte futurista di piacere. Sintesi di tecniche di seduzione, Cuneo, Nerosubianco Edizioni, 2011

61 - Meffre, Lilian, Carl Einstein 1885-1940 : itinéraires d'une pensée moderne, Paris, Presses de l'Université de Paris-Sorbonne, 2002

62 - Miretti, Lorenza "Manifesto e romanzo. Generi a confronto nel primo Futurismo", Bollettino '900 - Electronic Newsletter of '900 Italian Literature, décembre 2001, n. 2, http:// www3.unibo.it/boll900/numeri/2001-ii/W-bol/Miretti/

63 - Id., Mafarka il futurista. Epos e avanguardia, Bologna, Gedit, 2005

64 - Morel, Jean-Pierre, Le Roman insupportable. L'Internationale littéraire et la France, 1920-1932, coll. « Bibliothèque des idées », Gallimard, 1986

65 - Id., "Montage, collage et discours romanesque dans les années vingt et trente », in Collage et montage au théâtre et dans les autres arts durant les années vingt, Denis Bablet éd., Lausanne, L'âge d'homme, coll. " Théâtre Années 20 », 1991

66 - Id., «La Notion de montage dans le débat sur l'expressionnisme allemand en 1938 », in Expressionnisme(s) et avant-garde, Isabelle Krzywkowski et Cécile Millot dir., Paris, Editions L'improviste, Collection « Les aéronautes de l'esprit », 2007

67 - Navarro Domínguez, Eloy (éd.), Ramón Gómez de la Serna y la novela. Nuevas Perspectivas, colección Arias Montano, no 94, Huelva, Universidad de Huelva, 2009

68 - Pedullà, Gianfranco, Il mercato delle idee. Giovanni Gentile e la Casa editrice Sansoni, Bologna, Il Mulino, 1986

69 - Pedullà, Walter, «Le otto anime della bomba futurista ", in Futurismo e letteratura. L'Illuminista. Rivista di cultura contemporanea, $\mathrm{n}^{\circ} 27$, settembre/dicembre 2009

70 - Pérez Firmat, Gustavo, Idle Fictions: The Hispanic Vanguard Novel, 1926-1934, Durham, Duke University Press, 1982

71 - Rabaté, Dominique, Le Roman français depuis 1900, Paris, PUF, 1998 
72 - Raimond, Michel, La Crise du roman des lendemains du Naturalisme aux années vingt, Paris, José Corti, 1966

73 - Ródenas de Moya, Domingo, Los espejos del novelista. Modernismo y autorreferencia en la novela vanguardista española, Barcelona, Ediciones Península, 1998

74 - Id., «Introducción », in Ramón Gómez de la Serna : El novelista, Madrid, Colección Austral, 2005, p. 11-66.

75 - Rodríguez Fischer, Ana, Prosa española de vanguardia, Madrid, Clásicos Castalia, 1999

76 - Rolet, Serge, «La prose russe au tournant du XXe siècle: interprétation zéro ou sacralisation du livre?", La Posture de l'herméneute. Essais sur l'interprétation dans la littérature, Alison Boulanger et Jessica Wilker (dir.), Paris, Classiques Garnier, coll. "Rencontres", 2012, p. 71-83.

77 - Rubio, Emmanuel, «Par-delà modernité et avant-garde : le roman en archipel », in Réinventer le roman dans les années vingt, Myriam Boucharenc et Emmanuel Rubio éd., Villeneuve d'Ascq, Presses de l'Université Charles-de-Gaulle - Lille 3, 2010, p. 9-31

78 - Rugg, Marilyn D., "The Figure of the Author in Gómez de la Serna's El novelista », in Anales de la Literatura Espanola Contemporanea 14,1-3 (1989), p. 143-159.

79 - Salsano, Roberto, Trittico futurista: Buzzi, Marinetti, Settimelli, Roma, Bulzoni, 2006

80 - Serrano, José Enrique, "The theory of the novel in Ramón Gómez de la Serna's The Novelist », in The Spanish avant-garde, Derek Harris (ed.), Manchester/New York 1994, p. 27-38

81 - Shiach, Morag (éd.), The Cambridge Companion to The Modernist Novel, Cambridge University Press, 2007

82 - Sobejano-Moran, Antonio, « Aspectos modernistas en El incongruente, de Ramón Gómez de la Serna », AIH, Actas XII (1995). Tomo IV, p. 268-274, consultable en ligne : http:// cvc.cervantes.es/literatura/aih/pdf/12/aih_12_4_002.pdf

83 - Somville, Léon, "Les Romans autobiographiques de Pierre Reverdy », Etudes littéraires, vol. 3, $\mathrm{n}^{\circ} 1,1970$, p. 21-45

84 - Spires, Robert C., « New Art, New Woman, Old Constructs: Gómez de la Serna, Pedro Salinas, and Vanguard Fiction », Modern Language Notes, 115,2 (2000), 205-23.

85 - Id., Transparent Simulacra. Spanish fiction, 1902-1926, Columbia, University of Missouri Press, 1988

86 - Sprengel, Peter, « Erzählformen », Geschichte der deutschsprachigen Literatur 1900-1918. Von der Jahrhundertwende bis zum Ende des Ersten Weltkriegs, München, Beck, 2004

87 - Steinbach Dietrich (éd.), Prosa des Expressionismus, Stuttgart. Klett. 1982

88 - Tadié, Jean-Yves, Le Récit poétique, Paris, Gallimard, 1978

89 - Vassevière, Maryse, Aragon romancier intertextuel, ou, Les pas de l'étranger, Paris, L'Harmattan, 2000

90 - Verdone, Mario, Prosa e critica futurista, Milano, Feltrinelli, 1973

91 - Weber, Luigi, Romanzi del movimento, romanzi in movimento. La narrativa del futurismo $e$ dintorni, Bologna, Transeuropea, 2010 